\title{
A Model for Slew Evaluation for On-Chip RC Interconnects using Gamma Distribution Function
}

\author{
R. Kar \\ VLSI Laboratory \\ Department of ECE \\ NIT Durgapur-713209
}

\author{
V. Maheshwari \\ VLSI Laboratory \\ Department of ECE \\ NIT Durgapur-713209
}

\author{
Ashis K. mal \\ VLSI Laboratory \\ Department of ECE \\ NIT Durgapur-713209
}

\author{
A.K.Bhattacharjee \\ VLSI Laboratory \\ Department of ECE \\ NIT Durgapur-713209
}

\begin{abstract}
As technology scales down, timing verification of digital integrated circuits becomes an extremely difficult task due to statistical variations in the gate and wire delays. Statistical timing analysis techniques are being developed to tackle this problem. The variations of critical dimensions in modern VLSI technologies lead to variability in interconnect performance that must be fully accounted for the timing verification. However, handling a multitude of inter-die/intra-die variations and assessing their impacts on circuit performances can dramatically complicate the timing analysis. For optimizations like physical synthesis and static timing analysis, efficient interconnect delay and slew computation is critical. Slew indicates the rate of change of input/output signals. Slew rate determines the ability of a device to handle the varying signals. Determination of slew rate to a good proximity is thus very much essential for efficient design of high speed CMOS integrated circuits as the increase in waveform slew directly enhances the delay of the interconnections. This work presents an accurate and efficient model to compute the slew metric of on-chip interconnect of high speed CMOS circuits. Our slew metric assumption is based on the Gamma Distribution Function. The gamma distribution is used to characterize the normalized homogeneous portion of the step response. For a generalized RC interconnect model, the stability of the Gamma Distribution model is guaranteed. The better accuracy is proved by comparing our approach with the established methods and SPICE results. It is shown that our approach could result in the error in slew calculation as low as $2 \%$ with lower value of driver resistance when compared with the SPICE results.
\end{abstract}

\section{Categories and Subject Descriptors}

B.7.2 [Integrated Circuits]: Design Aids - Simulation;

\section{General Terms \\ Algorithms, Design, Theory}

\section{Keywords}

Moment Matching, On-Chip Interconnect, Probability Distribution Function, Slew calculation, Gamma Distribution, VLSI.

\section{INTRODUCTION}

Complex integrated systems on a single chip require communication between several components on the chip. Wires, buses or complex networks are used to transmit signals between subsystems. Interconnect delay computation is a critical task, which may be executed millions of times during floorplannig, placement, routing etc. So efficient, highly accurate and closedform delay and slew metrics are very important for IC designs. Modern chip designs contain an overwhelmingly large number of interconnects that must be analyzed efficiently. As such, efficiency of interconnect analysis is critical in a statistical timing flow. The advances in technology that result in scaled, multi-level interconnects may address the wire ability problem, but in the process create problems with signal integrity and interconnect delay. Elmore [1] proposed the impulse response of a linear circuit as a probability distribution function (PDF), using the mean of the impulse response to approximate the $50 \%$ delay of the circuit, which is the median of the impulse response under the probability interpretation under a step excitation. The Elmore delay metric has been incredibly popular because it is simple, closed-form, and easy to evaluate. However with development of the technology, interconnect delay is becoming comparable in value to cell delay or even dominates it, so in order to analyze the high speed VLSI circuit a priory, much more accurate interconnect delay and slew metrics are desired. AWE [5] can approach towards SPICE-like accuracy by computing and matching higher order moments of the impulse response, but AWE does not provide any closed-form formula, in particular it involves finding a solution of a non-linear equation. So a new delay metric is desired which should be highly accurate but also simple and closed-form.

As the technology is shrinking towards the ultra deep sub micrometer regime and transistor density in the chip is increasing, the length of the interconnect is getting longer [3]. So, efficient and accurate computation of slew metric is crucial for enhancing the switching speed of nano devices. In the nanotechnology age, as ultra deep sub-micron effects continue to wreak havoc on the integrity of the signal, so efficient and accurate computation of the slew metric has become critical.

In this paper we present a closed form slew metrics based on the Gamma distribution. Matching the circuit characteristics to that of the parameters of gamma distribution function produces an explicit closed form expression for slew calculation. Our approach is different with respect to the proposals made in [1] [12] in that our slew calculation does not require any look-up table. We have proposed the slew metric, GSM (Gamma Slew Metric) using the first two moments of the impulse response. The effectiveness and accuracy of the Gamma metric is justified on nets from an industrial design. We have compared with [13] which is based on Weibull distribution and found that our new approach is more 
accurate in capturing and estimating the slew metric for RC global interconnect.

\section{BASIC THEORY}

\subsection{Moments of a Linear Circuit Response}

Applying a Taylor series expansion of $e^{-s t}$ about $\mathrm{s}=0$ yields [7],

$$
\left.\begin{array}{rl}
H(s) & =\int_{0}^{\infty} h(t)\left\{1-s t+\frac{1}{2 !} s^{2} t^{2}-\frac{1}{3 !} s^{3} t^{3}+\ldots\right\} d t \\
& =\sum_{i=0}^{\infty} \frac{(-1)^{i}}{i !} s^{i} \int_{0}^{\infty} t^{i} h(t) d t
\end{array}\right\}
$$

The $i^{\text {th }}$ circuit-response moment, $\widetilde{m}_{i}$ is defined as [5]:

$\tilde{m}_{i}=\frac{(-1)^{i}}{i !} \int_{0}^{\infty} t^{i} h(t) d t$

From (2) and (3), the transfer function $\mathrm{H}$ (s) can be expressed as:

$H(s)=\widetilde{m}_{0}+\widetilde{m}_{1} s+\tilde{m}_{2} s^{2}+\tilde{m}_{3} s^{3}+\ldots$

\subsection{Central Moments}

Similar to moments, central moments are distribution theory concepts. Following Elmore's distribution function analogy, we can use them to explain the properties of Elmore delay approximation.

Consider the moment definition given in again:

$\widetilde{m}_{q}=\frac{(-1)^{q}}{q !} \int_{0}^{\infty} t^{q} h(t) d t$

The mean of the impulse response is given by [7] [12],

$\mu=\frac{\int_{0}^{\infty} \operatorname{th}(t) d t}{\int_{0}^{\infty} h(t) d t}=\frac{-m_{1}}{m_{0}}$

It is straightforward to show that the first few central moments can be expressed in terms of circuit moments as follows [6]:

$$
\left.\begin{array}{l}
\mu_{0}=m_{0} \\
\mu_{1}=0 \\
\mu_{2}=2 m_{2}-\frac{m_{1}^{2}}{m_{0}} \\
\mu_{3}=-6 m_{3}+6 \frac{m_{1} m_{2}}{m_{0}}-2 \frac{m_{1}^{3}}{m_{0}^{2}}
\end{array}\right\}
$$

Unlike the moments of the impulse response, the central moments have geometrical interpretations:

$\mu_{0}$ is the area under the curve. It is generally unity, or else a simple scaling factor is applied. $\mu_{2}$ is the variance of the distribution which measures the spread or the dispersion of the curve from the center. A larger variance reflects a larger spread of the curve.

$\mu_{3}$ is a measure of the skewness of the distribution; for a unimodal function its sign determines whether the mode (global maximum) is to the left or to the right of the expected value (mean). Its magnitude is a measure of the distance between the mode and the mean.

\subsection{Higher Central Moments in RC Tress}

The second and third central moments are always positive for RC tree impulse responses [6]. The positiveness of the second order central moment is obvious from its definition

$$
\mu_{2}=\int_{0}^{\infty}(t-\mu)^{2} h(t) d t
$$

The impulse response, $h(t)$, at any node in an RC tree is always positive. Hence the second central moment $\mu_{2}$ is always positive.

\subsection{Moments of Probability Density Function}

A probability function is a real valued set function where the domain is a subset of the sample space, $S$, and the range is a real number in the interval $[0,1]$. Generally, a function $P_{r}\{*\}$ should satisfy the three Kolmogorov axioms [8] (A), or equivalent conditions, in order to be considered as a probability function:

$$
\left.\begin{array}{l}
\text { (i) } P_{r}\{S\}=1 ; \\
\text { (ii) } P_{r}\{A\} \geq 0 \text { for all } A \in S \\
\text { (iii) } P_{r}\{A \cup B\}=P_{r}\{A\}+P_{r}\{B\} \\
\text { if } A \cap B=\varphi, A \in S, B \in S
\end{array}\right\}
$$

The distribution function of a continuous random variable $T$ denoted by $F_{T}(t)$ provides the value of $P_{r}\{T \leq t\}$ for any real number $-\infty \leq t \leq \infty$. The associated probability density function (PDF), denoted by $f_{T}(t)$ is the derivative of the distribution function with respect to $t$, thus

$$
\left.\begin{array}{rl}
f_{T}(t) & =\frac{d F_{T}(t)}{d t} \\
\text { and } F_{T}(t) & =\int_{-\infty}^{t} f_{T}(\tau) d \tau
\end{array}\right\}
$$

The median, $t(0.5)$, is defined by:

$$
P_{r}\left\{T \leq t_{(0.5)}\right\}=F_{T}\left(t_{(0.5)}\right)=\int_{-\infty}^{t_{(0.5)}} f_{T}(t) d t=0.5
$$

Whereas, the expected value or mean, $E(t)$ of a continuous random variable $T$ with distribution $f_{T}(t)$ is:

$$
E[T]=\int_{-\infty}^{\infty} t f_{T}(t) d t
$$

The mean is also the first moment of the distribution (or PDF). In general, the $i^{\text {th }}$ moment $m_{i}$ of the distribution is: 
$m_{i}=E\left[T^{i}\right]=\int_{-\infty}^{\infty} t^{i} f_{T}(t) d t$

Figure-1 summarizes these distribution definitions. Note that we use ' $\sim$ ' to distinguish between the probability moments $m_{1}, m_{2}$ and circuit moments $\widetilde{m}_{1}, \widetilde{m}_{2}$

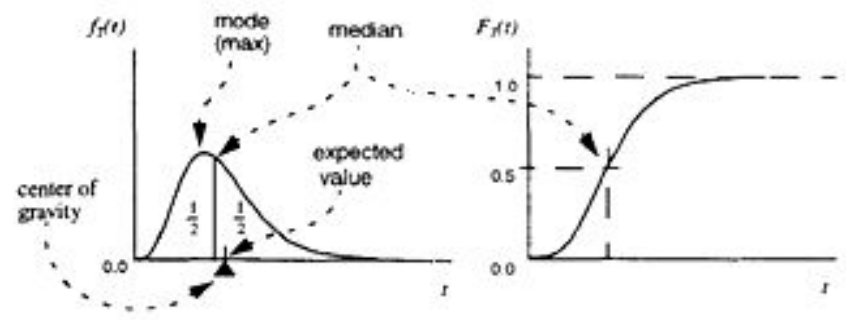

\subsection{Relation between Probability Density Functions and Circuit Response}

Any function $f(t)$ can be treated as a probability density function if it is defined in the range $[a, b]$ and satisfies [12]

$$
\left.\begin{array}{l}
f(t) \geq 0 \forall t \\
\int_{a}^{b} f(t) d t=1
\end{array}\right)
$$

If $f(t)$ is equal to zero outside of the range $[\mathrm{a}, \mathrm{b}]$, we can replace the integration limits in (13) with $-\infty$ and $\infty$. Elmore [1] was the first to apply moments for delay approximation of a limited class of circuit responses by observing that the impulse response of a circuit can be treated as a probability density function. $\mathrm{He}$ used this observation to justify the approximation of the $50 \%$ point of a monotonic step response (the median point of the impulse response) by the first moment (mean of the impulse response). It was shown that the impulse response corresponding to an RC tree is unimodal with positive skew [6]. From this it

Figure 1. A Probability density function ( left) and corresponding distribution function ( right)

follows that the mode is less than the median which is less than the mean and vice versa [8-9]:

\section{i.e. (Skew $>0)$ if and only if (mode $<$ median $<$ mean)}

Especially for the interconnects associated with deep submicron technologies, more than one moment is needed to capture the waveform shape-characteristics.

\section{PROPERTIES OF GAMMA DISTRIBUTION FUNCTION}

Elmore's original delay approximation is based on the analogy between non-negative impulse responses and probability density functions. In theory, Elmore's distribution interpretation can be extended beyond simply estimating the median by the mean if higher order moments can be used to characterize a representative distribution function. Once characterized, the delay can be approximated via closed form expression or table lookup of the median value for the representative distribution family. One proposal was to use a gamma distribution function [4]. The gamma distribution is a reasonably good representation of RC tree impulse responses since it provides good "coverage" of bell shaped curves which are bounded on the left and exponentially decaying to the right [10]. The Gamma distribution is depicted in Figure (2) [11]. The probability density function of gamma distribution $g_{\lambda, n}(t)$, is a function of one variable $t$ and two parameters $\lambda$ and $n$ (positive real numbers) [11]

$g_{\lambda, n}(t)=\frac{\lambda^{n} t^{n-1} e^{-\lambda t}}{\Gamma(n)}, t>0$

Where, $\Gamma(x)$ is the gamma function defined as:

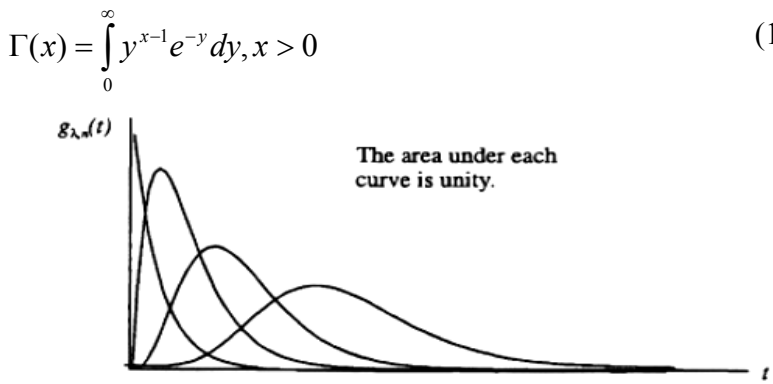

Figure 2. The Gamma Distribution Function

Now consider an impulse response $h(t)$ and assume that it is approximated with a gamma probability density function

$h(t)=g_{\lambda, n}(t)$

Then the transfer function is given by:

$H(s)=\left(\frac{\lambda}{\lambda+s}\right)^{n}$

The s-domain model denoted by (17) may be interpreted as a unique pole with a real number order. Notice that when $n=1$, the gamma distribution model naturally degrades to the dominant pole model. However, the existence of the parameter $n$ increases the degree of freedom of the model. The first few moments of the transfer function can be expressed easily in terms of the parameters $n$ and $\lambda[2]$.

$$
\left.\begin{array}{l}
m_{0}=1, \\
m_{1}=-\frac{n}{\lambda}, \\
m_{2}=\frac{n(n+1)}{2 \lambda^{2}}, \\
m_{3}=\frac{n(n+1)(n+2)}{6 \lambda^{2}}
\end{array}\right\}
$$

Since the gamma function has only two variables, it can be uniquely characterized by fitting it with two moments [2], [11]. For example, using the second and third equations, the parameters 
$\lambda$ and $n$ can be obtained from the first and second order circuit moments as:

$$
\left.\begin{array}{l}
\lambda=\frac{m_{1}}{m_{1}^{2}-2 m_{2}}, \\
n=\frac{-m_{1}^{2}}{m_{1}^{2}-2 m_{2}}
\end{array}\right\}
$$

In terms of the second central moment becomes

$$
\left.\begin{array}{r}
\lambda=\frac{-m_{1}}{\mu_{2}} \\
\text { and } \quad n=\frac{m_{1}^{2}}{\mu_{2}}
\end{array}\right\}
$$

Recall that both $-m_{1}$ (the Elmore delay) and $\mu_{2}$ are positive numbers for RC trees. Therefore the impulse response approximation with the parameters is always stable.

In the approximation above, we have used the first two moments. However, at least three moments are generally required to capture essential waveform response characteristics. Therefore, to match the third moment and capture the skewness of the distribution, we add a third variable $\Delta$, to include one more degree of freedom. We shift the gamma function $\Delta$ by to approximate the impulse response:

$$
h(t)=g_{\lambda, n}(t-\Delta)
$$

Thus the transfer function becomes

$$
H(s)=e^{-s \Delta}\left(\frac{\lambda}{\lambda+s}\right)^{n}
$$

and its moments are given by,

$$
\left.\begin{array}{l}
m_{1}=-\frac{n}{\lambda}-\Delta \\
m_{2}=\frac{n(n+1)}{2 \lambda^{2}}+\frac{n}{\lambda} \Delta+\frac{\Delta^{2}}{2} \\
m_{3}=-\frac{n(n+1)(n+2)}{6 \lambda^{3}}-\frac{n(n+1)}{2 \lambda^{2}} \Delta-\frac{n}{\lambda} \frac{\Delta^{2}}{2}-\frac{\Delta^{3}}{6}
\end{array}\right\}
$$

with $m_{0}=1$. We now have three unknowns, $\lambda, n$ and $\Delta$ in three equations. It can be shown that this equation.

system can also be expressed in terms of $m_{1}$ and second and third central moments, $\mu_{2}$ and $\mu_{3}$.

$$
\left.\begin{array}{l}
m_{1}=-\frac{n}{\lambda}-\Delta \\
\mu_{2}=-\frac{n(n+1)}{\lambda^{2}}-\left(\frac{n}{\lambda}\right)^{2}=\frac{n}{\lambda^{2}} \\
\mu_{3}=\frac{n(n+1)(n+2)}{\lambda^{3}}-3\left(\frac{n}{\lambda}\right) \frac{n(n+1)}{\lambda^{2}}+2\left(\frac{n}{\lambda}\right)^{3}=\frac{2 n}{\lambda^{3}}
\end{array}\right\}
$$

Note that the second and third order central moments $\mu_{2}$ and $\mu_{3}$ are independent of the shift $\Delta . \lambda$ and $n$ are the measures of variance and skewness of the waveform, respectively, and they are not affected by the time shift. This results,

$$
\begin{aligned}
& \lambda=\frac{2 \mu_{2}}{\mu_{3}} \\
& n=\frac{4 \mu_{2}^{3}}{\mu_{3}^{2}}
\end{aligned}
$$

$\Delta=-m_{1}-\frac{n}{\lambda}$

We now find an expression for the step response, $y(t)$, which is the integral of the impulse response,

$$
y(t)=\int_{0}^{t} h(\tau) d \tau
$$

After some algebraic manipulation we obtain,

$$
y(t)=\int_{0}^{\lambda(t-\Delta)} g_{\lambda, n}(\tau) d \tau=P(n, \lambda(t-\Delta))
$$

Where $P(n, x)$ is the incomplete gamma function.

$$
P(n, \lambda)=\frac{1}{\Gamma(n)} \int_{0}^{t} x^{n-1} e^{-x} d x
$$

Where $\Gamma(n)$ is the gamma function. Note that $P(n, t)$ is zero at $\mathrm{t}=$ 0 and monotonically increases to one. Hence, to calculate the delay at a particular percentage point we only need to find the value of $x$ such that

$P(n, x)=\alpha$

Where $x=\lambda(t-\Delta)$. Then with a simple scaling and shifting, the delay is obtained:

$t_{\alpha}=\frac{x}{\lambda}+\Delta$

\subsection{Calculation of Parameters of the Gamma Distribution function}

Since gamma has only two parameters $\lambda$ and $n$ matching two moments would completely characterize this model. Hence Mean of the Gamma function is given by

$\frac{n}{\lambda}=-m_{1}$

Variance of the Gamma function is given by

$\frac{n}{\lambda^{2}}=-\left(m_{1}^{2}-2 m_{2}\right)$

From (31) and (32),

$\left.\begin{array}{rl}\lambda & =\frac{m_{1}}{m_{1}^{2}-2 m_{2}} \\ \text { and } \quad n=\frac{-m_{1}^{2}}{m_{1}^{2}-2 m_{2}}\end{array}\right\}$ 
In terms of the second central moment becomes [from (19)]

$$
\lambda=\frac{-m_{1}}{\mu_{2}}, n=\frac{m_{1}^{2}}{\mu_{2}}
$$

Recall that both $-m_{1}$ (the Elmore delay) and $\mu_{2}$ are positive numbers for RC trees. Therefore the impulse response approximation with the parameters is always stable.

\section{PROPOSED CLOSED FORM SLEW METRIC}

The step response of an RC circuit is a cumulative density function (CDF) [7]. The RC response is considered as a singlepole exponential waveform and can be modeled as

$h(t)=1-e^{-\frac{t}{\lambda}}, t>0$, If $\mathrm{h}$ (t) satisfies the following conditions:

$0 \leq h(t) \leq 1$ and $\operatorname{Lim}_{t \rightarrow-\infty} f(t)=0, \underset{t \rightarrow \infty}{\operatorname{Lim}} f(t)=1$

Now, let $T_{L O}$ and $T_{H I}$ be $10 \%$ and $90 \%$ delay points, respectively. Matching to these points to the CDF yields

$$
\begin{aligned}
& 0.1=1-e^{-\frac{T_{L O}}{\lambda}} \\
& 0.9=1-e^{-\frac{T_{H I}}{\lambda}}
\end{aligned}
$$

From equations (35) \& (36), we have

$$
\begin{aligned}
& T_{L O}=\lambda \ln \left(\frac{10}{9}\right)=0.1053 \lambda \\
& T_{H I}=\lambda \ln (10)=2.302 \lambda
\end{aligned}
$$

Using equations (37) and (38), we define the gamma slew metric (we call this metric as GSM) as

$$
G S M=T_{H I}-T_{L O}=2.1976 \lambda
$$

Using equation (39), we can write the closed form expression of the slew metric in terms of first two circuit moments as

$$
G S M=\frac{2.1967 m_{1}}{m_{1}^{2}-2 m_{2}}
$$

From the above derived equation (40) for the Slew Metric for the on-chip interconnect using Gamma Distribution Function; we see that the Slew Metric function is a simple function of the two first circuit moments. This is our proposed closed form slew model.

\section{EXPERIMENTAL RESULTS}

In order to verify the efficiency of our model, we have extracted 208 routed nets containing 1026 sinks from an industrial ASIC design in $0.18 \mu \mathrm{m}$ technology. We choose the nets so that the maximum sink delay is at least $10 \mathrm{ps}$ and the delay ratio between closet and furthest sinks in the net is less than 0.2 . It ensures that each net has at least one near end sink. We classify the 1026 sinks into the following three categories:

513 far-end sinks have delay greater or equal to $75 \%$ of the maximum delay to the furthest sink in the net.

342 mid-end sinks which have delay between $25 \%$ and $75 \%$ of the maximum delay and,

171 near-end sinks which have delay less than or equal to $25 \%$ of the maximum delay.

For each sink we compute the slew using SPICE simulator. We compare our slew metric with [1], [12] and [13]. We call these metrics as EDS, BakS and WbS, respectively. The comparison of our slew metric (GSM) with BakS, WbS and EDS is shown in Table I-IV. From the results shown in table I-IV, we find that our proposed model provides the best slew estimation compared to other approaches and results an average error of less than $2 \%$ for lower value of driver resistance and an average $6 \%$ for higher value of driver resistance.

\begin{tabular}{|l|l|l|l|l|}
\hline sinks & BaKS & EDS & WbS & GSM \\
\hline Near & 65.45 & 786.13 & 43.72 & 39.34 \\
\hline Mid & 11.76 & 24.27 & 4.65 & 3.91 \\
\hline Far & 9.23 & 11.23 & 2.831 & 1.987 \\
\hline Total & 9.23 & 11.23 & 2.831 & 1.987 \\
\hline
\end{tabular}

Table 1. Average (\%) relative error with Diver resistance $=$ $0 \Omega$

\begin{tabular}{|l|l|l|l|l|}
\hline sinks & BaKS & EDS & WbS & GSM \\
\hline Near & 17.25 & 143.23 & 15.34 & 11.54 \\
\hline Mid & 12.3 & 31.2 & 7.87 & 6.59 \\
\hline Far & 9.45 & 16.6 & 6.78 & 5.69 \\
\hline Total & 10.35 & 29.4 & 7.23 & 5.98 \\
\hline
\end{tabular}

Table 2. Average (\%) relative error with Diver resistance $=100 \Omega$ 
Table 3. \% standard deviation with driver resistance $=0 \Omega$

\begin{tabular}{|l|l|l|l|l|}
\hline sinks & BaKS & EDS & WbS & GSM \\
\hline Near & 44.25 & 615.1 & 27.38 & 19.63 \\
\hline Mid & 7.832 & 23.62 & 4.59 & 3.79 \\
\hline Far & 6.96 & 10.30 & 3.1 & 2.78 \\
\hline Total & 6.96 & 10.30 & 3.1 & 2.78 \\
\hline
\end{tabular}

Table 4. \% standard deviation with driver resistance $=100 \Omega$

\begin{tabular}{|l|l|l|l|l|}
\hline sinks & BaKS & EDS & WbS & GSM \\
\hline Near & 18.3 & 98.9 & 15.66 & 12.32 \\
\hline Mid & 10.23 & 26.56 & 7.67 & 6.71 \\
\hline Far & 7.12 & 78.65 & 6.98 & 5.91 \\
\hline Total & 10.54 & 78.65 & 6.98 & 5.86 \\
\hline
\end{tabular}

\section{CONCLUSION}

We have proposed Gamma Distribution function based closed form Slew Metric model for the RC trees that is a simple function of two moments of impulse response. Our model has Elmore delay as upper bound but with significantly less error. The novelty of our approach is justified by the calculated slew from the experiments performed on the industrial nets. For a generalized $\mathrm{RC}$ interconnect model the stability of the homogeneous Gamma Distribution model is guaranteed.

\section{REFERENCES}

[1] Elmore, W.C., "The transient response of damped Linear network with Particular regard to Wideband Amplifiers", J. Applied Physics, 19, 1948, pp.55-63.

[2] Kay, R., Pileggi, L. PRIMO: Probability Interpretation of Moments for Delay Calculation, IEEWACM Design Automation Conference, 1998, pp. 463-468.

[3] Yang Wu, Shien, Boon-Khim Liew, K.L. Young, C.H.Yu, and S.C. Analysis of Interconnect Delay for $0.18 \mu \mathrm{m}$ Technology and Beyond" IEEE International Conference Interconnect Technology, May 1999 , pp. 68 - 70

[4] Lin, T. , Acar, E. , Pileggi, L., h-gamma: An RC Delay Metric Based on a Gamma Distribution Approximation to the Homogeneous Response, IEEE/ACM International Conference on Computer-Aided Design, 1998, pp. 19-25.

[5] Pillage, L. T., Rohrer, R. A., Asymptotic Waveform Evaluation for Timing Analysis, Tran. on CAD, Volume 9, Issue 4, 1990. pp. 331- 349

[6] Gupta, R. , Tutuianu, B., Pileggi, L. T. , The Elmore Delay as a Bound for RC Trees with Generalized Input Signals, IEEE Trans. on CAD, 16(1), pp. 95-104, 1997.

[7] Mustafa Celik, Lawrence Pileggi, Alten Odabasioglu, "IC Interconnect Analysis", Kluwer Academic Publishers, 2002

[8] M. G. Kendall and A. Stuart, "The Advanced Theory of Statistics, vol. 1: Distribution Theory", New York: Hafner, 1969.

[9] MacGillivray, H. L , The Mean, Median, Mode Inequality and Skewness for a Class of Densities, Australian J. of Statistics, vol. 23, Issue 2, 1981 Pages 247-250.

[10] Chu, C. , Horowitz, M. "Charge-Sharing Models for Switch-Level Simulation", Volume 6, Issue 6, November 1987, pp.:1053 - 1061.

[11] Harald Cramer. Mathematical Methods of Statistics. Princeton University Press, 1946.

[12] Bakoglu, H. B., Circuits, Interconnects, and Packaging for VLSI. Addison-Wesley Publishing Company, 1990.

[13] Kar, R.; A..K. Mal, A. K. Bhattacharjee, "An Accurate Slew Metric for on-chip VLSI Interconnect using Weibull Distribution Function " ACM ICAC3 2009. 601-604 January 2009. 